\title{
QUELQUES ÉLÉMENTS DU SIGNIFIÉ DE MADĪNA: L'EMPLOI DE KA CHEZ AL-IDRT̃ST
}

\author{
Por \\ DRA. CHRISTINE MAZZOLI-GUINTARD \\ Universidad de Caen
}

La terminologie employée par les textes arabes médiévaux pose, aux historiens, bien des probłèmes; curieusement d'ailleurs, ce n'est pas tant le contenu à mettre sous le signifiant qui donne lieu à des débats, mais plutôt la manière d'utiliser la terminologie. Certains, tel M. de Epalza, insistent sur l'importance à accorder au vocabulaire puisque «les Arabes appellent chaque chose par son nom»; il faut donc «reconnaître et respecter ce contenu sémantique fondamental de la langue arabe, sans quoi les réalités se déforment» (1). D'autres au contraire estiment que les termes qui apparaissent dans les sources écrites n'ont pas de contours nettement définis; selon M. Acién Almansa, par exemple, «la langue des sources n'est absolument pas précise» (2).

II nous semble toutefois que ces deux perceptions de la terminologie utilisée par les textes arabes médiévaux, loin de se contredire, se complètent: d'un côté, en effet, une approche purement philologique d'un document écrit, c'està-dire une lecture qui considère le texte lui-même comme étant un objet de connaissance, cette approche intrinsèque au document permet sans aucun doute de saisir, en partie au moins, le signifié des termes employés. D'un autre côté, une approche plus historique d'un document écrit, qui vise en particulier à saisir les structures d'un peuplement attache plus d'importance à la façon dont le peuplement est décrit, tente de mettre cette description en rapporte avec la

(1) M. de Epalza: «Precisiones sobre instituciones musulmanas de las Baleares», $V$ Jornades d'Estudis Històrics Locals, Palma de Mallorca, 1987, 73-87, en particulier p. 84.

(2) M. Acién Almansa: «Poblamiento y fortificación en el sur de al-Andalus. La formación de un país de husun», II Congreso de Arqueología Medieval Española, Oviedo, 1989, I, 137-149. 
réalité archéologique. Or, chaque méthode, à notre avis, saisit une partie différen de la réalité: la première fournit une première indication intéressante, puisqu'elle précise comment l'objet caché sous le signifiant est à la fois conçu et perçu par les hommes qui utilisent le concept; la seconde méthode, s'appuyant sur les résultats de la première, permet ensuite d'essayer d'appréhender la réalité matérielle de l'objet. Ainsi, dans une première démarche, interroger un texte d'un point de vue strictement philologique, permet d'avoir, d'un objet historique, une première vision pouvant servir à étayer une réflexion.

Pourquoi ne pas en revenir à la structure fondamentale de la société islamique: la ville? Les historiens s'accordent en général pour reconnaître dans madina le terme qui désigne le plus couramment le centre urbain (3): est-il possible, à travers la lecture intrinsèque d'un texte, de saisir quel sens lui donnent les auteurs arabes?

A cet égard, le texte d'al-Idrīsi mérite une attention particuliére: l'oeuvre de ce géographe du Xllème siècle, même si elle puise ses renseignements à des sources de natures diverses (4), présente une certaine unité de rédaction.

De plus, dans la partie consacrée à al-Andalus, le terme madina revient fréquemment et semble faire référence à une unité de peuplement que l'auteur distingue de hişn et de qarya: ainsi, «la province d'Alpujarras [compte] parmi ses villes (mudun), Jaén, un grand nombre de châteaux (huşunn) et plus de six cents villages (qurā)» (5). Ces trois concepts classificatoires du mode de peuplement, madina, hişn et qarya, que l'on traduit avec parfois trop d'empressement par ville, château et village, méritent en réalité une approche plus nuancée: P. Guichard montre comment, en ce qui concerne la région de Valence, madinna renvoie essentiellemnt à l'idée de centre de district, à quel point hișn fait principalement référence à un site fortifié de plus ou moins forte importance et qui structure le peuplement, tandis que qarya, forme d'habitat dotée elleaussi bien souvent d'une fortification, se trouve davantage dépendante d'une autre localité (6).

Enfin, et surtout, le texte d'Al-Idrīs livre au lecteur une série de phrases nominales mettant en comparaison madina et un autre terme, hiṣn mais aussi qarya, au moyen d'une préposition invariable, ka (comme, semblable à) (7). Comparaison n'est certes pas raison, mais essayer de comprendre pourquoi un rapport est établi entre deux objets linguistiques peut fournir des indications sur le contenu de ces objets, et en particulier sur celui qui nous intéresse, madina.

(3) Voir par exemple R. Dozy: Recherches sur l'histoire et la littérature de l'Espagne pendant le Moyen Age. 3ème éd., Amsterdam, 1965, I, 300: madina recouvre le sens de ville, mais aussi ceux de capitale et de province.

(4) C.E. Dubler: “Los caminos a Compostela en la obra de IdrīsT̄», Al-Andalus XIV (1949), 59-122; "Las laderas del Pirineo según Idrīsī», idem, XVIII (1953), 337-373.

(5) Al-Idrīsī, Opus geographicum, fasc. V, Naples-Rome, 1975, 537.

(6) P. Guichard: Les Musulmans de Valence et la Reconquête (Xlè-Xllè siècles), I, Damas, 1990, 194-202.

(7) Sur son emploi, voir par exemple A. Périer: Nouvelle grammaire arabe, Paris, 1940, 264-265. 
Quelles sont donc ces expressions? Nous les reproduisons ici (8):

1.- «al-Andalus [...] wa-fī [...] ḥuṣūn kātîra ka-l-mudun ‘āmira» (9).

2.- «iqlīm Ǔsūna wa-fT-hi ḥuṣūn ‘āmira ka-l-mudun» (10). (11).

3.- «madīnat Turğālla kabīra (ka)-I-hișn al-manī wa-la-hā aswār manīà"

4.- «ḥiṣn Bukayrān ḥiṣn manī ‘āmir ka-J-madīna» (12).

5.- «ḥisn Š Saqūra ka-l-madīna ‘̄ämir» (13).

6.- «Biz[mi]liȳana [...] wa-hiya qarya ka-I-madina [...] wa-bi-hā al-hammam wa-l-fanadiq» (14).

7.- «Qǐsața wa-huwa ḥiṣn ka-l-madīna la-hu aswāq wa-rabạ̣ 'āmir wahammām wa-fainādiq» (15).

8- «ḥișn Qabra kabīr ka-l-madīna ḥașin al-makān wațTq al-binyān» (16).

Il est possible de les traduire ainsi:

1.- «al Andalus [comprend] [...] un foisonnement de châteaux peuplés comme des villes".

2.- «l'iqlim d'Osuna [comprend] des châteaux peuplés comme des villes».

3.- «Trujillo [est] une grande ville semblable à un château inexpugnable et ses murs [sont] solidement bâtis».

4.- «Bocairente est un château inexpugnable peuplé comme une ville».

5.- «Segura [est] un château peuplé comme une ville».

6.- «Bezmiliana [est] un village semblable à une ville, [...], pourvu d'un bain et de fondouks».

7.- «Quesada [est] un château semblable à une ville qui [possède] des souks, un faubourg peuplé, des bains et des fondouks».

8.- «Cabra [est] un grand château semblable à une ville, fortifié et solidement construit».

Il est possible de décomposer chaque phrase de la manière suivante: la préposition ka articule deux termes -que nous désignons par commodité

(8) Al-Idrîsi Opus geographicum, fasc. V, Naples-Rome, 1975. Nous tenons à exprimer ici notre gratitu de enver Ma J. Viguera pour l'aide précieuse qu'elle nous a apportée dans la translittération de ce document.

(9) íbidem, p. 536

(10) libidem, p. 537.

(11) ibidem, p. 550.

(12) libidem, p. 557

(13) Ibidem, p. 560.

(14) libidem, p. 565.

(15) libidem, p. 569.

(16) íbidem, p. 571 


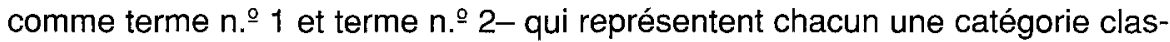
sificatoire de peuplement. Dans la plupart des séquences retenues, le terme $n$. o 2 reçoit un complément dans lequel l'on peut chercher des éléments permettant d'expliquer le motif du rapport établi avec le terme $n .^{\circ} 1$. Plus rarement, c'est dans le terme $n . \stackrel{1}{1}$, dans la plus grande proximité possible de la particule qu'il faut essayer de comprendre ce qui permet de rapprocher les deux termes. Ainsi, l'on peut estimer que le motif du rapport établi entre le terme $n . \stackrel{\circ}{1}$ et le terme $n .22$ de la comparaison constitue un élément du signifié du second terme; par exemple, lorsqu'il est dit qu'une ville est semblable à un château inexpugnable, le caractère imprenable est bien un trait qui définit le château.

Si maintenant l'on rapproche de façon schématique les séquences, on obtient les résultats suivants:

Le signifié de madinna chez al-Idr̄̄̄ī apparaît immédiatement associé à une notion relativement abstraite, celle d'cāmir (17). D'un autre côté, le terme madīna doit être mis en relation avec des objets concrets: hammām, fanādiq, aswāq et rabad (18). Enfin, la dernière donnée du signifié se trouve dans la notion de wațqq al-binyān (19).

La madinna d'al-Idr̄is̄̄ correspond bien, d'un côté, à l'image traditionnelle de la ville: l'on y trouve en effet les éléments indispensables à la vie quotidienne, marchés, bains et fondouks. II faut tout de suite noter l'absence, dans cette énumération des bâtiments essentiels de la madina, d'une structure de caratère religieux: la mosquée constitue sans doute une donnée tellement intrinsèque à tout peuplement islamique qu'il n'est même plus nécessaire d'en signaler la présence.

Par contre, la madina se caractérise par la présence de deux structures indispensables à la vie économique: le marché et le fondouk. La première indique la présence de liens entre le centre urbain et une périphérie dont il est difficile de préciser l'étendue: les souks ruraux hebdomadaires, selon P. Chalmeta

\begin{tabular}{|c|c|c|c|}
\hline séquence $n . ?$ & 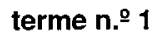 & terme $\mathrm{n} . \mathbf{2} 2$ & motif du rapport établi \\
\hline $\begin{array}{l}1 \\
2 \\
3 \\
4 \\
5 \\
6 \\
7 \\
- \\
8\end{array}$ & $\begin{array}{l}\text { ḥusūn } \\
\text { ḥusūn } \\
\text { madīna } \\
\text { hișn } \\
\text { ḥișn } \\
\text { qarya } \\
\text { hiș̣n } \\
\text { hișn }\end{array}$ & $\begin{array}{l}\text { mudun } \\
\text { mudun } \\
\text { h̦ișn } \\
\text { madina } \\
\text { madína } \\
\text { madina } \\
\text { madina } \\
\text { madīna }\end{array}$ & 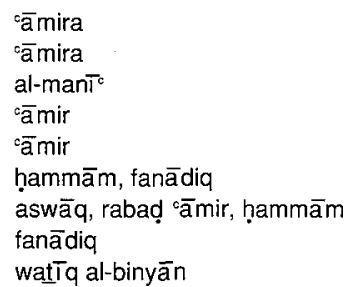 \\
\hline
\end{tabular}

(17) Séquences n. $91,2,4,5,7$. Voir, sur ce terme, M. de Epalza, «Estudio del texto de al-Idrisi sobre Alicante", Sharq al-Andalus, 2, 1985, pp. 219-220.

(18) Séquences $n .96$ et 7 .

(19) Séquences $n . ? 3$ et 8. 
Gendrón, attirent les populations qui se trouvent dans un rayon géographique correspondant à un jour de marche (20). Il est bien difficile d'aller au-delà d'hypothèses semblables pour les souks urbains permanents. La seconde structure, quant à elle, suppose l'existence de relations commerciales à plus longue distance: il s'agit en effet d'un bâtiment qui sert à la fois d'entrepôt pour les marchands (21); parfois même, certains fondouks sont destinés exclusivament à des marchands étrangers. À Valence, par exemple, les Pisans et les Gênois reçoivent un fondouk; une structure identique est signalée à Almería, à Denia, mais aussi à Séville ou à Grenade (22).

La présence du bain, associée au concept de madinna, ne surprend guère: elle est traditionnellement mise en relation avec des prescriptions religieuses (23). Mais cette vision classique ne doit pas faire oublier que le bain suppose également la présence d'un peuplement relativement important; en effet, comme le démontre fort bien A. Raymond, que le bain soit un bien waqf, fondé au profit d'une oeuvre pie, ou une propriété dépendant d'un particulier qui en attend des revenus, son existence dépend entièrement de son utilisation par le public (24); autrement dit, le bain n'existe que s'il est rentable, ce qui se réalise s'il est fréquenté.

Ainsi, la madina, chez al-IdrTsi, se caractérise-t-elle tout d'abord par des structures concrètes qui rattachent le concept à une notion de centre économique, peut-être lié à des échanges à longue ou moyenne distance, et à une idée de peuplement relativement dense.

Mais au-delà, cette madinna contient aussi l'idée d'un espace qui est '⿳亠̄mmir, c'est-à-dire un lieu habité, peuplé, à l'état de culture, qui s'opose à ce qui est sauvage, ruiné ou abandonné, mais aussi un endroit prospère et florissant, bien pourvu (25).

Cette conception de la madina n'est pas sans rappeler l'image de la ville qui transparaît dans les écrits d'Ibn Haldūn: la contradiction fondamentale de la société se trouve dans l'opposition qui existe entre la ville et la bādiya, et plus particulièrement même entre la ville et deux types spécifiques de la bāadiya, celle des nomades et des montagnards, surtout d'ailleurs ceux qui habitent des montagnes dont l'accès est difficile (26). Pourquoi une telle opposition? Essentiellement parce que nomades et montagnards demeurent dans des

(20) P.Chalmeta Gendrón: El señor del zoco en España, Madrid, 1973.

(21) L. Torres Balbás: «Las alhóndigas hispanomusulmanas y el Corral del Carbón de Granada», AlAndalus, XI (1946), 447-480.

(22) ídem.

(23) Voir par exemple Grupo de estudio «urbanismo musulmán», Baños árabes en el país valenciano, Generalitat Valenciana, 1989.

(24) A. Raymond: "Signes urbains et étude de la population des grandes villes arabes à l'époque ottomane», Bulletin d'Etudes Orientales, n. . XXVII (1974), 183-193. A la prêsence d'une population relativement nombreuse, l'on peut rattacher l'existence du faubourg (rabad).

(25) A. de Biberstein Kazimirski: Dictionnaire arabe-français, Paris, 1960, 11, 365.

(26) A. Oumlil: «Ibn Khaldoun et la société urbaine», dans A. Bouhdiba et D. Chevallier: La ville arabe dans I'slam, histoire et mutations, Tunis, 1982, 39-44. 
zones isolées, aux conditions d'existence souvent très rudes. Suivre jusqu'au bout Ibn Haldūn dans son raisonnment représente une tentation à laquelle il est bien difficile de résister: les deux types de la bādiya qui forment l'opposé même de la ville finissent par constituer des groupes «solidement structurés par de fortes cohésions de clans nécessitées par le besoin élémentaire de la survie et de la défense» (27). La notion d'‘āmir étroitement associée au concept de madina manifeste donc bien l'opposition qui existe entre deux modes de peuplement distincts, mais qui coexistent.

Le concept d'cāmir doit également être rapproché de l'idée de culture: sur la racine 'amara, en effet, se forme 'imāra, la civilisation (28). C'est dans la madina, essentiellement que les ulémas viennent enseigner ou apprendre: la fréquence des mentions de villes, dans les dictionnaires biographiques, bien étudiée pour la région orientale d'al-Andalus (29), met en valeur l'existence de centres fondamentaux de la vie culturelle, tels Valence, Murcie, Játiva et Denia. Même si l'analyse plus fine de ces données pose un certain nombre de problèmes, les villes principales du Sarq al-Andalus correspondent bien à des capitales culturelles. En effet, la ville islamique, comme l'écrit M. Marín, «n'est pas seulement l'endroit où le musulman peut accomplir ses devoirs religieux et ses idéaux sociaux, mais aussi l'endroit où la culture islamique se transmet» (30).

Chez al-Idrīsī, donc, madīna comporte l'idée d'un espace qui a abandonné l'état de nature pour celui de culture, aussi bien d'ailleurs celui de cultivo que celui de cultura.

Enfin, la madina d'al-Idrīsī représente aussi une structure wațiq al-binyān, c'est-à-dire une construction solide, inébranlable (31). Il s'agit, d'une part, d'un espace sans doute bien bâti, aux murailles robustes, à propos duquel surgit bien rapidement le concept de fortification, de château; or, non seulement al-idrīs̄̄ a tendance à comparer mađīna et hişn, mais encore les auteurs arabes.confondent-ils bien souvent, dans leur discours, hişn et madina: Madrid, par exemple, apparaît tantôt comme un hişn, tantôt comme une madinna dans les textes recensés par Ma J. Viguera (32), sans qu'il soit possible d'y déceler, par exemple, une évolution du site. Ibn Hayyān l'évoque comme un hișn à l'époque de Muhammad I, puis, en 939-940, comme une madina; Ibn cidāir la qualifie de madinna en 977 , et de hișn en 1197; al-Idrīsī, sensiblement à la même époque y fait référence comme étant une madına; Ibn Haldūn la signale, à propos d'événements survenus dans le dernier quart du XIIlème siècle, comme un hişn (33).

(27) ĺdem, 40.

(28) R. Dozy: Suppléments aux dictionnaires arabes, Paris-Leyde, 2ème éd,, 1927, II, 170-172.

(29) P. Guichard: Les Musulmans de Valence et la Reconquête (Xlè-XIllè siècles), I, Damas, 1990, 192 et document $n .936$.

(30) M. Marín: "Ciencia, enseñanza y cultura en la ciudad islámica», Simposio internacional sobre la ciudad islámica, Zaragoza, 1991, 113-133, en particulier p. 120.

(31) A. de Biberstein Kazimirski: Dictionnaire arabe-français, Paris, 1960, II, 1485: waț $7 q$ ou ferme, solide, inébranlable, bien cimenté.

(32) Ma J. Viguera: «Madrid en al-Andalus», II Jarique de Numismática Hispano-Arabe, Madrid, 1990, sous presse (Viguera, "Madrid").

(33) idem. 
La situation escarpée de Madrid -quelle que soit l'hypothèse de reconstitution du site à l'époque islamique que l'on retienne (34) - à côte de son rôle de centre de district -lbn Hayyān y signale la présence de gouverneurs (35)- expliquent sans doute en partie la confusion des auteurs, confusion qui ne doit plus surprendre, puisque les signifiés des deux termes se recouvrent en partie, au moins dans l'idée de fortification.

Cependant, au-dèlà d'un espace bien fortifié, que l'on peut aisément confondre avec un hișn, la notion de wafīq al-binyān sous-entend aussi que derrière la madina se cachent des moyens financiers, techniques, humains qui rendent possible une telle solidité de la construction. Possédons-nous quelques indices qui nous permettent de cerner ces moyens? Le cas de Badajoz représente un exemple dont l'on ne peut assurer qu'il soit vraiment significatif et représentatif, mais qui possède l'avantage d'être bien connu (36); l'on y observe comment un rebelle en rupture de ban, Ibn Marwān al-GilliqT, fonde une ville et obtient d'cAbd Allăh une sorte de reconnaissance politique sur la région qu'il domine. Ce qui, dans cette affaire, est remarquable, c'est qu'lbn Marwān demande à l'émir «qu'on lui envoyât des ouvriers chargés de construire la mosquée et les bains» (37). On ignore les motifs d'une telle requête; est-on en droit de supposer qu'ils sont financiers, que la bourse de l'Etat omeyyade est mieux garnie que celle d'lbn Marwān... Cela ne ferait que renforcer l'idée haldūnienne selon laquelle la ville est liée à l'Etat (38); la mise en place d'une structure de peuplement urbain passe-t-elle nécessairement par l'Etat? C'est la question qui peut surgir face à cette notion de wațiq al-binyān qu'al-Idrīsī associe à celle de madina.

Comment conclure? S'attacher à la lecture intrinsèque d'un document ne semble pas dénué d'intérêt: l'étude de la préposition ka, utilisée avec madina para al-ldrīst,laisse apparaître un objet linguistique qui revêt, chez cet auteur, des contours relativement précis (39). Madrna correspond de la sorte à un ensemble de structures concrètes qui en font un centre où s'effectuent des echanges à moyenne ou longue distance, mais aussi un centre assez densément peuplé; mađ̄ina, espace 'āmir, espace fortifié, doit être dotée de moyens suffisants pour lui assurer une construction solide.

Toutefois il convient de ne pas oublier les limites de cette étude: elle se borne non seulement à un auteur, mais encore à un aspect de son discours,

(34) Voir les hypothèses de J. Oliver Asin: Historia del nombre Madrid, Madrid, 1952, 2. ${ }^{2}$ ed. Madrid, 1991, introducción de M. ${ }^{\hat{z}} \mathrm{~J}$. Rubiera Mata; de B. Pavón Maldonado: "Arqueología y urbanismo medieval en Madrid. De la Almudayna árabe a la torre mudéjar de San Nicolás", Awrāq, núms. 7-8, 1984-1985, pp. 231-278; de F.J. Marín Perellón: «Las murallas árabes de Madrid», /l Congreso de Arqueología Medieval Española, Madrid, 1987, II, 744-754; de F. Valdés Fernández: «El Madrid islámico. Notas para una discusión arqueológica», Madrid, castillo famoso, Madrid, 1990, 127-158.

(35) Viguera: «Madrid».

(36) C. Picard: «La fondation de Badajoz por “Abd al-Raḥmān ibn Yūnus al-JillikT̄ (fin IXè siècle)", Revue d'Etudes Islamiques, XLIX,2 (1981), 215-229.

(37) Ídem, 219.

(38) A. Oumlil: «lbn Khaldoun et la société urbaine», dans A. Bouhdiba et D. Chevallier: La ville arabe dans IIslam histoire et mutations, Tunis, 1982, 39-44, et en particulier p. 41.

(39) On ne peut manquer d'être frappé par le fait que, dans cinq cas sur huit, madinna est associée à l'idée d'c̄àmir. 
celui qui utilise la préposition ka; nous ignorons, d'autre part, si certains aspects du signifié de mad̄na sont suffisants. Il est certain, par exemple, que l'idée de peuplement, à elle-seule, n'est absolument pas apte, chez al-Idrīsî, à définir la madinna; à propos de Ségovie, al-Idrīsis'exprime en effet de la façon suivante: "Ségovie n'est pas une ville, mais beaucoup de villages proches les uns des autres, à tel point qu'ils se touchent, et ses habitants, nombreux et bien organisés [...] possèdent de grands pâturages» (40). Enfin, cette étude n'a aucune autre prétention que de tenter de comprendre comment al-Idrīsi pense la notion de madina et d'essayer de montrer que cette conception revêt dans son discours une certaine cohérence. Cela signifie, entre autres, que de la conclusion de P. Guichard concernant les difficultés posées par la terminologie des sources arabes, nous préférons la seconde hypothèse à la première; il écrit en effet: "comme il arrive souvent, la terminologie arabe est peu précise, et nous ne savons d'ailleurs pas quel niveau de connaissance les auteurs des trop rares textes à notre disposition avaient des réalités concrètes auxquelles ils font allusion» (41). C'est cette seconde donnée qu'il nous semble fondamental de conserver sans cesse présente à l'esprit puiqu'elle ne peut que nous inciter à nous méfier de conclusions hâtives. Mais cela signifie, également, que vouloir comprendre comment l'idée de madinna, conçue et imaginée à la cour de Roger II de Sicile, peut s'appliquer à la réalité d'al-Andalus, nécessite un travail bien plus long et semé d'embûches que cette étude qui ne peut rien fournir d'autre que des indications de recherche; ce travail suppose en effet une enquête archéologique, inséparable d'un retour, ensuite, vers la documentation textuelle (42): méthode plus fructueuse, a priori, qu'une recherche de caractère monolithique, trop rigide, mais méthode aussi d'emploi plus complexe (43).

(40) Al-IdrīsT: Geografía de España, traduction par E. Saavedra: Textos Medievales, n. ${ }^{\circ}$ 37, Valencia, 1974, 145-146.

(41) P. Guichard: Les Musuimans de Valence et la Reconquête (Xlè-XIlè siècle), I, Damas, 1990, 202.

(42) Cette méthode de travail est exposée par exemple chez A. Debord: "Castrum et Castellum chez Adémar de Chabannes", Archéologie mediévale, IX (1979), 97-109.

(43) Voir les difficultés posés dans P. Guichard: „Depuis Valence et en allant vers l'ouest, bilan et propositions pour une équipe», Mélanges de la Casa de Velázquez, XXVI-1 (1990), 163-195, en particulier p. 184-188. 